\title{
Metastasiertes Nierenzellkarzinom Verbessertes Überleben durch zielgerichtete Therapie
}

\begin{abstract}
Der Multikinase-Inhibitor Sunitinib führt bei Patienten mit metastasiertem Nierenzellkarzinom (mRCC) im Vergleich zu den bisher verfügbaren Behandlungsoptionen zu einer Verbesserung der objektiven Ansprechrate und des progressionsfreien Überlebens.
\end{abstract}

Durch den Einsatz zielgerichteter Therapien kann heute neben der Lebensqualität auch das Überleben von Patienten mit mRCC verbessert werden. Das entscheidende Kriterium für das Therapiemanagement sei der Start mit einer hoch effektiven Therapie, berichtete Kurt Miller, Berlin. Dabei seien neben den Überlebensdaten vor allem die Ansprechraten ein wichtiges Entscheidungskriterium in der Erstlinientherapie. Ein hohes Ansprechen ermögliche eine rasche Reduktion tumorassoziierter Beschwerden und motiviere den Patienten zur Therapiefortsetzung, so Miller. Zudem zeigen erste Daten auch bei mRCC einen Zusammenhang zwischen Qualität des Tumoransprechens und dem Gesamtüberleben (OS) [Krajewski K et al. Eur Urol. 2011;59:856-62].

Sunitinib $\left(\right.$ Sutent ${ }^{\circledR}$ ) bewirkt ein besonders hohes Tumoransprechen und gute Überlebensdaten. Daher wird die Substanz als Therapie der Wahl in der Erstlinientherapie von Patienten mit guter und mittlerer Prognose empfohlen [Miller K et al. Aktuel Urol. 2011;42:242-6]. In der Zulassungsstudie vervierfachte Sunitinib die objektive Ansprechrate gegenüber Interferon-alpha (IFN; 47 vs. $12 \%, \mathrm{p}<0,0001)$. Gleichzeitig wurde das progressionsfreie Überleben unter Sunitinib mehr als verdoppelt (median 11 vs. 5 Monate, $\mathrm{p}<0,001)$. Das OS betrug im Sunitinib-Arm 26,4
Monate gegenüber 21,8 Monaten im IFN-Arm (Hazard Ratio 0,818; $\mathrm{p}=0,049$ ) [Motzer RJ et al. J Clin Oncol. 2009;27:3584-90].

Mittlerweile liegen Erfahrungen mit Sunitinib über fünf Jahre vor. Auch Patienten mit gastrointestinalen Stromatumoren (GIST), bei denen eine Therapie mit Imatinib fehlgeschlagen ist oder nicht vertragen wurde, können von Sunitinib profitieren - einschließlich Patienten mit Exon-11- und Exon9-Mutationen [Heinrich MC et al. J Clin Oncol. 2008;26;5352-9]. Weiterhin ist die Substanz zur Therapie nicht resezierbarer oder metastasierten pankreatischen neuroendokrinen Tumoren mit Krankheitsprogression zugelassen.

ami

Fachpressegespräch „5 Jahre SunitinibTherapie - Eine Standortbestimmung" anlässlich der 8. AIO-Herbsttagung; 17. November 2011 in Berlin; Veranstalter: Pfizer Pharma

\section{Gastrointastinale Stromatumoren Adjuvante Therapie für drei Jahre empfohlen}

Das wissenschaftliche Beratergremium CHMP der europäischen Arzneimittelbehörde EMA hat die Empfehlung ausgesprochen, eine dreijährige Behandlung mit Imatinib bei gastrointestinalen Stromatumoren in die Fachinformation aufzunehmen.

Die Indikation für eine dreijährige Therapie mit Imatinib (Glivec $^{\circledast}$ ) gilt für Patienten, bei denen ein C-KIT (CD117)-positiver Tumor reseziert worden ist und die ein hohes Rezidivrisiko aufweisen. Imatinib ist bereits seit 2009 zur Therapie dieser Stromatumoren zugelassen. Allerdings waren die Patienten in der zulassungsrelevanten Studie nur zwölf Monate behandelt worden.

Basis für die beantragte Änderung der Fachinformation sind aktuelle Daten der Phase-III-
Studie „SSG XVIII/AIO“. Darin wurden die Ergebnisse einer adjuvanten Therapie mit $400 \mathrm{mg}$ Imatinib pro Tag über drei Jahre mit denen einer einjährigen Behandlung verglichen. Einschlusskriterien waren u. a. Patienten mit hohem Rezidivrisiko:

1. Tumorgröße $>5 \mathrm{~cm}$, Mitoserate $>5$ pro 50 High-Power-Fields (HPF) in der Mikroskopie,

2. Tumorgröße $>10 \mathrm{~cm}$ und beliebige Mitoserate,

3. beliebige Tumorgröße und Mitoserate $>10$ pro $50 \mathrm{HPF}$ und
4. Tumorruptur im peritonealen Raum.

Nach einer Beobachtungsdauer von fünf Jahren waren $65,6 \%$ der Patienten in der Gruppe mit dreijähriger Behandlung rezidivfrei; nach einjähriger Behandlung waren es nur 47,9\%. 92,0\% der Teilnehmer aus dem Studienarm mit drei Jahren Behandlung waren noch am Leben, während die Überlebensrate nach einem Jahr Therapie bei $81,7 \%$ lag. Das Risiko, innerhalb des fünfjährigen Beobachtungszeitraums zu sterben wurde durch die dreijährige adjuvante Therapie gegenüber der einjährigen halbiert.

Nach Informationen von Novartis Pharma 